\title{
Diagnostic Fine-Needle Biopsy of Small Solid Pancreatic Lesions Using a Franseen Needle during Endoscopic Ultrasound Examination
}

\author{
Kosuke Takahashi (D), Ichiro Yasuda *(D), Tatsuyuki Hanaoka, Yuka Hayashi, Yasuhiro Araki, Iori Motoo, \\ Shinya Kajiura, Takayuki Ando, Haruka Fujinami, Kazuto Tajiri (D, Masami Minemura and Terumi Takahara
}

check for

updates

Citation: Takahashi, K.; Yasuda, I.; Hanaoka, T.; Hayashi, Y.; Araki, Y.; Motoo, I.; Kajiura, S.; Ando, T.;

Fujinami, H.; Tajiri, K.; et al.

Diagnostic Fine-Needle Biopsy of Small Solid Pancreatic Lesions Using a Franseen Needle during Endoscopic Ultrasound Examination. Diagnostics 2021, 11, 27. https://dx.doi.org/ 10.3390/diagnostics11010027

Received: 13 November 2020 Accepted: 23 December 2020 Published: 25 December 2020

Publisher's Note: MDPI stays neutral with regard to jurisdictional claims in published maps and institutional affiliations.

Copyright: () 2020 by the authors. Licensee MDPI, Basel, Switzerland. This article is an open access article distributed under the terms and conditions of the Creative Commons Attribution (CC BY) license (https: / / creativecommons.org/ licenses/by/4.0/).
Third Department of Internal Medicine, University of Toyama, Toyama, 2630 Sugitani, Toyama 930-0194, Japan; ktakaako@med.u-toyama.ac.jp (K.T.); T.Hanaoka.com@outlook.jp (T.H.); yukaberry0822@yahoo.co.jp (Y.H.); aajtpx5830@yahoo.co.jp (Y.A.); iori4869@med.u-toyama.ac.jp (I.M.); shin-ya@nsknet.or.jp (S.K.); taando33@gmail.com (T.A.); haruka52@med.u-toyama.ac.jp (H.F.); tajikazu@med.u-toyama.ac.jp (K.T.); minemura@med.u-toyama.ac.jp (M.M.); taka@med.u-toyama.ac.jp (T.T.)

* Correspondence: yasudaic@med.u-toyama.ac.jp

\begin{abstract}
Background and aim: During endoscopic ultrasound-guided fine needle aspiration biopsy (EUS-FNB), Franseen needles can help collect sufficient tissue to permit histopathological assessment. However, its efficacy might be limited by the size of the targeted lesion. This study aimed to evaluate the feasibility of histopathological assessment of small solid pancreatic lesions using a 22-gauge Franseen needle during EUS-FNB. Methods: This retrospective study evaluated data from all patients who underwent EUS-FNB using a Franseen needle for solid pancreatic lesions at the University of Toyama Hospital between June 2018 and April 2020. Results: The study included 159 patients who had 152 malignant lesions and 7 benign lesions. The malignant lesions included pancreatic cancers $(n=134)$, neuroendocrine neoplasms $(n=15)$, metastatic tumors $(n=2)$, and a solid pseudopapillary neoplasm $(n=1)$. The diagnostic accuracy of EUS-FNB (combining histology and cytology) was $98.7 \%$. However, the histopathological diagnosis was only confirmed for $64.3 \%$ of small lesions ( $<10 \mathrm{~mm}$ ), relative to $97.2 \%$ for larger lesions. Multivariate analysis also revealed that lesion size of $<10 \mathrm{~mm}$ predicted a less accurate histopathological diagnosis (odds ratio: $6.97,95 \%$ confidence interval: $1.02-47.67 ; p=0.041$ ). Further analyses revealed a failed histological diagnosis in 4 patients with lesions of $<5 \mathrm{~mm}$ in size and accurate diagnoses in 9 out of 10 patients with lesions of $5-10 \mathrm{~mm}$ in size. Conclusions: The diagnostic accuracy for small lesions $(<10 \mathrm{~mm})$, especially for lesions of $<5 \mathrm{~mm}$, based on histological examination alone, was significantly lower than that for others $(>10 \mathrm{~mm})$. Furthermore, multivariate analysis revealed that only lesion size was an independent predictor of histopathological diagnosis accuracy.
\end{abstract}

Keywords: EUS-FNB; Franseen needle; histopathological assessment; small pancreatic lesions

\section{Introduction}

Endoscopic ultrasound-guided fine needle aspiration (EUS-FNA) is the gold standard technique for diagnosing solid pancreatic masses. The pathological assessment was originally based on cytological findings, which provide $85-97 \%$ sensitivity, $88-98 \%$ specificity, and 78-96\% diagnostic accuracy [1-3]. However, histopathological assessments provide more information regarding tissue architecture and immunohistological staining, thus facilitating a more accurate and precise diagnosis than that obtainable with cytological assessment [4]. In addition, histopathological findings, including immunostaining, are essential for diagnosing various diseases, such as lymphoma, autoimmune pancreatitis, and other rare tumors. Large-caliber 19-gauge needles have been used to obtain sufficient biopsy samples for histopathological assessment [5-7] although this technique has some technical issues related to the stiff shaft and unsharpened tip. To overcome these issues, 
third-generation FNA needles, including Franseen needles, were recently developed [8]. Franseen needles have three symmetric heels at the tip and their unique shape helps obtain a sufficient sample for histopathological assessment in most cases, even if the needle is a conventional caliber (22-gauge, 22G). Several studies have indicated that using a Franseen needle during EUS-guided fine needle biopsy (EUS-FNB) provides various benefits [9-11], although the feasibility of using Franseen needles might be limited for smaller lesions. Therefore, this study aimed to evaluate the feasibility of histopathological assessment for small pancreatic lesions using a 22G Franseen needle during EUS-FNB.

\section{Materials and Methods}

\subsection{Patients}

This retrospective single-center study was conducted at the University of Toyama Hospital and included all patients who underwent EUS-FNB for solid pancreatic lesions between June 2018 and April 2020. During this period, a 22G Franseen needle (Acquire; Boston Scientific, Marlborough, MA, USA) was routinely used as the first biopsy needle at our institution. The patient and lesion characteristics, final diagnosis, diagnostic ability, and adverse events of EUS-FNB using a 22G Franseen needle were investigated. The severity of adverse events was defined according to the American Society for Gastrointestinal Endoscopy classification [12]. This retrospective study protocol was approved by the institutional review board of our institution (R2020096) on 25 August 2020, and registered in the University Hospital Medical Information Network clinical trials registry (UMIN000041511). All patients had provided informed consent for the EUS-FNB procedure.

\subsection{Procedural Technique}

The EUS was performed as an in-patient procedure using a curved linear echoendoscope (GF-UCT260; Olympus Corporation, Tokyo, Japan) connected to an ultrasound scanning system (EU-ME2; Olympus Corporation, Tokyo, Japan). The patients underwent EUS-FNB under conscious sedation with midazolam, and their vital signs were monitored. Two experienced endosonographers (I.Y. and K.T.) performed the EUS-FNB using a 22G Franseen needle (Acquire; Boston Scientific) in all cases, as previously reported [13]. The lesion was punctured via the stomach or duodenum under guidance from real-time EUS imaging, and color Doppler imaging was used to confirm that the puncture path would not disrupt any major vessels or the main pancreatic duct. The stylet was then removed, a $20 \mathrm{~mL}$ syringe was attached to the needle, and $10 \mathrm{~mL}$ of negative pressure was applied. Several movements were made within the lesion and the suction was slowly released after the movements were completed. The needle was then withdrawn into the sheath and the entire system was withdrawn from the biopsy channel. The aspirated material was expelled onto glass slides by carefully reinserting the stylet into the needle. All patients were observed for $\geq 24 \mathrm{~h}$ after the procedure.

The specimen was macroscopically evaluated, and the whitish portions (macroscopically visible core) were collected and placed on a small piece of filter paper. The sample was then placed in formalin solution for histological examination and the remaining material was smeared on glass slides for cytological examination. As our institution does not have an on-site pathologist or cytologist, punctures were repeated up to three times until a whitish material was macroscopically observed. If the third puncture was not successful, the lesion was diagnosed based on only the cytological examination.

\subsection{Definition}

In this study, the diagnosis based on the EUS-FNB specimen was determined using the histological and cytological findings. The "histological diagnosis" only considered the histological findings (without cytology) and included pancreatic cancer, neuroendocrine neoplasm (NEN), metastatic tumor, and solid pseudopapillary neoplasm (SPN). The cytological diagnosis was classified as definite, suspected malignancy (including NEN and $\mathrm{SPN}$ ), or benign, based on the Bethesda system. Cases judged as "definite" or "suspicious" 
by cytological diagnosis were defined as malignant. The final diagnosis was based on: (1) definite evidence of malignancy from a surgical specimen, (2) a diagnosis of malignancy based on the EUS-FNB findings and clinical/imaging follow-up compatible with malignant disease, or (3) no evidence of malignancy based on the EUS-FNB findings and clinical/imaging follow-up of $\geq 6$ months.

\subsection{Endpoints}

The primary endpoint was the diagnostic accuracy of EUS-FNB for small pancreatic lesions (diameter of $<10 \mathrm{~mm}$ ). The diagnostic histopathological results of small pancreatic lesions were compared with those of other lesions $(>10 \mathrm{~mm})$. We also analyzed factors that were associated with the accuracy of the histopathological diagnosis using EUS-FNB.

\subsection{Statistical Analyses}

Continuous variables were presented as median (range) and categorical variables were presented as number (percentage). Univariate and multivariate analyses were performed to identify the factors influencing the accuracy of the histopathological diagnosis using EUS-FNB. The univariate analyses were performed using the chi-squared test or Fisher's exact test for categorical variables and the Mann-Whitney U-test for continuous variables. The multivariate analysis was performed using a logistic regression model, and factors with a univariate $p$-value of $<0.05$ were entered into the multivariate model. All analyses were performed using JMP ${ }^{\circledR}$ software (version 15; SAS Institute, Inc., Cary, NC, USA).

\section{Results}

During the study period, 159 patients underwent EUS-FNB for pancreatic lesions, using a $22 \mathrm{G}$ Franseen needle. The baseline patient and lesion characteristics are shown in Table 1. The patients included 94 men and 65 women, and the median age was 71 years (range: $34-89$ years). The median lesion size (largest diameter) was $28.4 \mathrm{~mm}$ (range: $4.2-76.2 \mathrm{~mm}$ ), and 14 lesions were $<10 \mathrm{~mm}$. The puncture route was through the stomach in 101 cases, through the D1 segment (duodenal bulb) in 36 cases, and through the D2 segment (second part of the duodenum) in 22 cases. The median number of needle passes was 2 (range: 1-3). No adverse events were associated with the EUS-FNB. The cytological diagnoses were of definite malignancy in 121 cases, suspected malignancy in 28 cases, and benign in 10 cases. The histological diagnoses were of adenocarcinoma in 133 cases, NEN in 10 cases, SPN in 1 case, and no malignancy in 15 cases (Table 2).

Table 1. Baseline characteristics of the patients and the targeted lesions.

\begin{tabular}{|c|c|}
\hline & $\mathbf{N}=159$ \\
\hline Age, years, median (range) & $71(34-89)$ \\
\hline Gender $(\mathrm{M} / \mathrm{F})$ & $94 / 65$ \\
\hline \multicolumn{2}{|l|}{ Lesions } \\
\hline Size, mm, median (range) & $28.4(4.2-76.2)$ \\
\hline \multicolumn{2}{|l|}{ Size } \\
\hline$<10 \mathrm{~mm} / 10-20 \mathrm{~mm} />20 \mathrm{~mm}$ & $14 / 36 / 109$ \\
\hline Main location of the lesion & \\
\hline Head/body/tail & $77 / 44 / 38$ \\
\hline
\end{tabular}

NEN, neuroendocrine neoplasm; SPN, solid pseudopapillary neoplasm. 
Table 2. Detailed data of endoscopic ultrasound-guided fine needle aspiration biopsy (EUS-FNB).

\begin{tabular}{cc}
\hline & $\mathbf{N}=\mathbf{1 5 9}$ \\
\hline Puncture route & \\
Transgastric & 101 \\
Trans D1 & 36 \\
Trans D2 & 22 \\
Number of needle passes, median (range) & $2(1-3)$ \\
FNB diagnosis (cytology) & 121 \\
Malignant (definitive) & 28 \\
Malignant (suspicious) & 10 \\
Benign & 133 \\
FNB diagnosis (histology) & 10 \\
Adenocarcinoma & 1 \\
NEN & 15 \\
SPN & 0 \\
No malignancy &
\end{tabular}

The diagnosis based on EUS-FNB was of malignancy in 150 cases and no malignancy in 9 cases. The diagnoses of 150 cases that were considered to have malignancy based on the EUS-FNB were confirmed to be malignancy based on the surgical specimen (77 cases) or based on the clinical follow-up (73 cases). Among the 9 cases that were judged to not be malignant based on the EUS-FNB, 7 cases involved benign lesions based on the clinical follow-up, 1 patient was diagnosed with a malignant lesion after re-examination of the EUS-FNB findings, and 1 patient was diagnosed with duodenal invasion of pancreatic cancer based on findings from endoscopic biopsy and the surgical specimen. Thus, the final diagnoses were of malignant lesions in 152 cases and benign lesions in 7 cases (Figure 1). The malignant lesions included 134 pancreatic cancers, 15 NENs (13 cases with grade 1 NENs and 2 cases with grade 2 NENs), 2 metastatic tumors from lung cancer, and 1 SPN (Table 3).

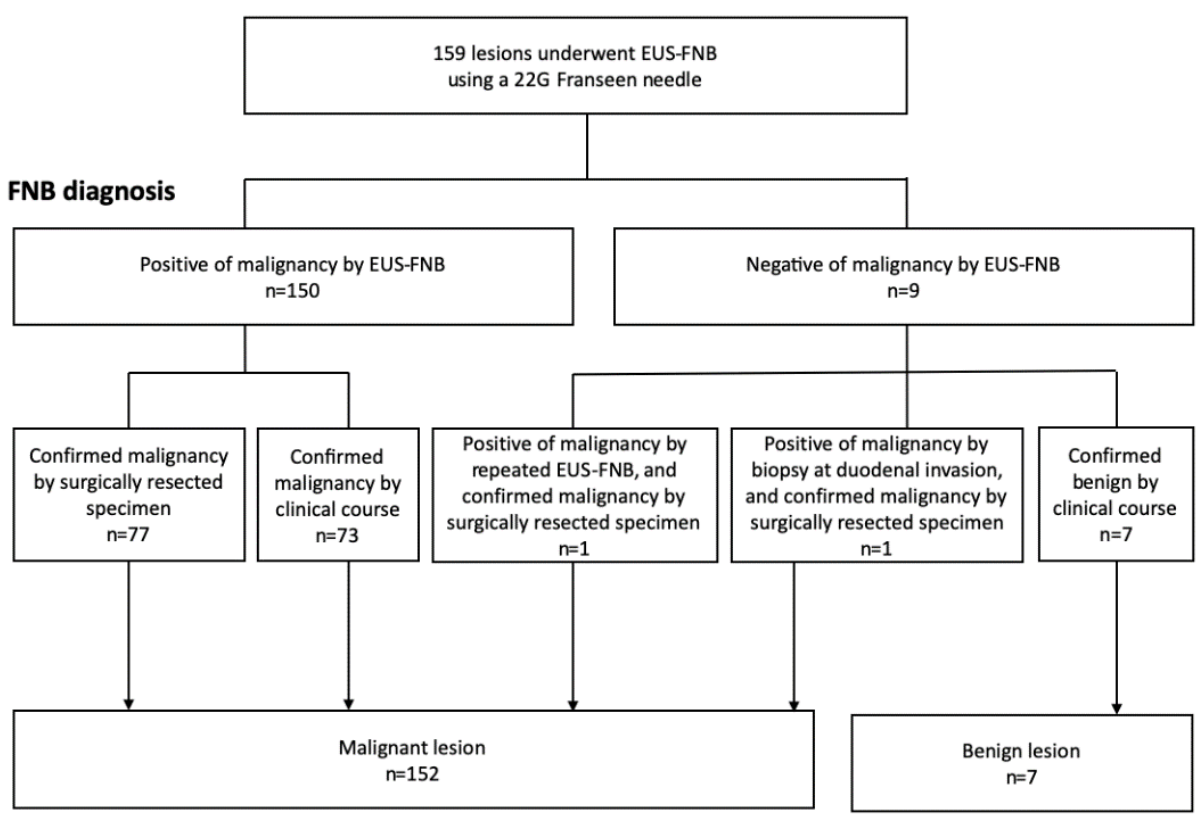

Final diagnosis

Figure 1. Flowchart of diagnosis via endoscopic ultrasound-guided fine-needle biopsy (EUS-FNB). ERCP, endoscopic retrograde cholangiopancreatography. 
Table 3. Final diagnosis of the patients who underwent EUS-FNB.

\begin{tabular}{cc}
\hline & $\mathbf{N}=\mathbf{1 5 9}$ \\
\hline Pancreatic cancer & 134 \\
NEN (NET G1/G2) & $15(13 / 2)$ \\
Metastatic tumor & $2 *$ \\
SPN & 1 \\
Focal chronic pancreatitis & 7 \\
\hline
\end{tabular}

NEN, neuroendocrine neoplasm; SPN, solid pseudopapillary neoplasm. * metastasis of lung cancer.

\subsection{Diagnostic Results of EUS-FNB}

The diagnostic results of EUS-FNB are shown in Table 4. The EUS-FNB procedure (combining histology and cytology) provided 98.7\% accuracy, 98.7\% sensitivity, 100\% specificity, a positive predictive value (PPV) of 100\%, and a negative predictive value (NPV) of $77.7 \%$. Based on the histological findings alone, the procedure provided $94.3 \%$ accuracy, $94.1 \%$ sensitivity, $100 \%$ specificity, a PPV of $100 \%$, and an NPV of $40 \%$. The accuracies of the histological findings were $64.3 \%$ for cases with lesions of $<10 \mathrm{~mm}$ and $97.2 \%$ for cases with lesions of $\geq 10 \mathrm{~mm}(p<0.001)$ (Table 5).

Table 4. Diagnostic results of EUS-FNB.

\begin{tabular}{cccccc}
\hline & Accuracy & Sensitivity & Specificity & PPV & NPV \\
\hline $\begin{array}{c}\text { Combined of histology } \\
\text { and cytology }\end{array}$ & $98.7 \%$ & $98.7 \%$ & $100 \%$ & $100 \%$ & $77.7 \%$ \\
Only cytology & $94.3 \%$ & $96.6 \%$ & $44.4 \%$ & $97.3 \%$ & $50.0 \%$ \\
Only histology & $94.3 \%$ & $94.1 \%$ & $100 \%$ & $100 \%$ & $40.0 \%$ \\
\hline
\end{tabular}

PPV, positive predictive value; NPV, negative predictive value.

Table 5. Diagnostic histological results of EUS-FNB.

\begin{tabular}{cccccc}
\hline & Accuracy & Sensitivity & Specificity & PPV & NPV \\
\hline$<10 \mathrm{~mm}$ & $64.3 \%$ a) & $64.3 \% \mathrm{~b})$ & $\mathrm{N} / \mathrm{A}^{\mathrm{c}}$ & $100 \%$ & $0 \%$ \\
$\geq 10 \mathrm{~mm}$ & $97.2 \%$ & $98.6 \%$ & $66.6 \%$ & $98.6 \%$ & $66.6 \%$ \\
\hline
\end{tabular}

PPV, positive predictive value; NPV, negative predictive value. ${ }^{\text {a) }} p<0.001 ;{ }^{\text {b) }} p<0.001$; ${ }^{\text {c) }}$ Specificity could not be calculated because there were no false-positive and true-negative cases.

\subsection{Factors Influencing the Accuracy of the Histopathological Diagnosis}

Factors influencing the accuracy of the histopathological diagnosis were evaluated using univariate and multivariate analyses (Table 6). Univariate analyses revealed that diagnostic accuracy was significantly associated with age of $\geq 70$ years, lesion size of $\geq 10 \mathrm{~mm}$, and final diagnosis of pancreatic cancer. However, the diagnostic accuracy was not significantly associated with sex, regular use of antithrombotic drugs, lesion location, serum carbohydrate antigen 19-9 level, or number of needle passes. The three significant variables were included in the multivariate model, which revealed that diagnostic accuracy was only independently associated with lesion size of $<10 \mathrm{~mm}$ (odds ratio: 6.97, 95\% confidence interval: $1.02-47.67, p=0.041$ ).

\subsection{Subgroup Analysis of Lesions that Were $<10 \mathrm{~mm}$}

The baseline characteristics and pathological outcomes of cases with lesions that were $<10 \mathrm{~mm}$ are shown in Table 7. The diagnosis based on EUS-FNB (combining histology and cytology) was accurate in 13 out of 14 cases, although the histopathological diagnosis was only accurate in 9 cases. In particular, a sufficient sample for the histopathological assessment could not be obtained in all 4 cases with lesions of $<5 \mathrm{~mm}$, which resulted in inaccurate diagnoses. However, the histopathological diagnoses were accurate in 9 out of 10 cases with lesions measuring in the range of 6-10 $\mathrm{mm}$. 
Table 6. Univariate and multivariate analyses of factors influencing the accurate histopathological diagnosis of EUS-FNB.

\begin{tabular}{|c|c|c|c|c|c|c|c|c|}
\hline \multirow{2}{*}{ Variable } & \multirow{2}{*}{$\mathbf{N}$} & \multirow{2}{*}{$\begin{array}{c}\text { Accurate Histopathological } \\
\text { Diagnosis (\%) }\end{array}$} & \multicolumn{3}{|c|}{ Univariate Analysis } & \multicolumn{3}{|c|}{ Multivariate Analysis } \\
\hline & & & OR & $95 \%$ CI & $p$-Value & OR & $95 \%$ CI & $p$-Value \\
\hline \multicolumn{9}{|l|}{ Age (years) } \\
\hline $70 \leq$ & 97 & 97.9 & 6.05 & $1.21-30.13$ & 0.029 & 2.56 & $0.52-12.50$ & 0.251 \\
\hline$<7 \overline{0}$ & 62 & 88.7 & & & & & & \\
\hline \multicolumn{9}{|l|}{ Gender } \\
\hline Female & 94 & 96.8 & 3.08 & $0.74-12.81$ & 0.161 & - & - & - \\
\hline \multirow{2}{*}{\multicolumn{9}{|c|}{ Antithrombotic drugs }} \\
\hline & & & & & & & & \\
\hline+ & 19 & 94.7 & 1.09 & $0.13-9.24$ & 1.000 & - & - & - \\
\hline- & 140 & 94.3 & & & & - & - & - \\
\hline \multicolumn{9}{|l|}{ Location } \\
\hline Body/tail & 82 & 97.6 & 4.00 & $0.80-19.89$ & 0.091 & - & - & - \\
\hline Head & 77 & 91.9 & & & & - & - & - \\
\hline \multicolumn{9}{|l|}{ Serum CA19-9 level } \\
\hline$\leq 37 \mathrm{U} / \mathrm{mL}$ & 53 & 90.6 & 0.37 & $0.10-1.47$ & 0.161 & & & \\
\hline$>37 \mathrm{U} / \mathrm{mL}$ & 106 & 96.2 & & & & & & \\
\hline \multicolumn{9}{|l|}{ Size of lesion } \\
\hline $10 \mathrm{~mm}<$ & 145 & 97.2 & 19.58 & $4.47-85.80$ & $<0.001$ & 6.97 & $1.02-47.67$ & 0.041 \\
\hline$<10 \mathrm{~mm}$ & 14 & 64.3 & & & & & & \\
\hline \multicolumn{9}{|c|}{ Number of needle passes } \\
\hline $1-2$ & 132 & 94.7 & 1.43 & $0.28-7.28$ & 0.650 & - & - & - \\
\hline 3 & 27 & 92.6 & & & & - & - & - \\
\hline \multicolumn{9}{|l|}{ Final diagnosis } \\
\hline Pancreatic cancer & 134 & 97.8 & 13.79 & $3.18-59.80$ & 0.001 & 3.87 & $0.53-28.03$ & 0.195 \\
\hline Other diseases & 25 & 76.0 & & & & & & \\
\hline
\end{tabular}

OR, odds ratio; $\mathrm{CI}$, confidence interval.

Table 7. Detailed results of EUS-FNB for lesions smaller than $10 \mathrm{~mm}$.

\begin{tabular}{cccccccc}
\hline & Age/Gender & Tumor Size, $\mathbf{m m}$ & Tumor Location & Puncture Site & Final Diagnosis & Cytology Result & Histology Result \\
\hline 1 & $62 / \mathrm{F}$ & 4.2 & Body & Stomach & NET (G1) & Susp. NEN & Inadequate \\
2 & $62 / \mathrm{F}$ & 4.2 & Body & Stomach & NET (G1) & Susp. NEN & Inadequate \\
3 & $67 / \mathrm{F}$ & 4.3 & Head & D1 & NET (G1) & Susp. NEN & Inadequate \\
4 & $67 / \mathrm{F}$ & 4.8 & Head & D1 & NET (G1) & Susp. NEN & Inadequate \\
5 & $86 / \mathrm{F}$ & 6.5 & Head & Stomach & NET (G1) & Benign & Inadequate \\
6 & $61 / \mathrm{F}$ & 6.6 & Body & Stomach & NET (G1) & Susp. NEN & NEN \\
7 & $67 / \mathrm{F}$ & 8.5 & Head & Stomach & PC & Adenoca. & Adenoca. \\
8 & $68 / \mathrm{M}$ & 8.8 & Head & D1 & PC & Adenoca. & Adenoca. \\
9 & $86 / \mathrm{F}$ & 8.8 & Tail & Stomach & NET (G1) & Susp. NEN & NET (G1) \\
10 & $69 / \mathrm{F}$ & 8.9 & Head & Stomach & NET (G1) & Susp. NEN & NET (G1) \\
11 & $78 / \mathrm{M}$ & 9.1 & Head & Stomach & PC & Adenoca. & Adenoca. \\
12 & $85 / \mathrm{M}$ & 9.1 & Body & Stomach & NET (G1) & Susp. NEN & NEN \\
13 & $34 / \mathrm{F}$ & 9.4 & Tail & Stomach & SPN & Susp. SPN & SPN \\
14 & $89 / \mathrm{M}$ & 9.9 & Tail & Stomach & NET (G1) & Susp. NEN & NET (G1) \\
\hline
\end{tabular}

D1, descending part of the duodenum; NET, neuroendocrine tumor; PC, pancreatic cancer; SPN, solid pseudopapillary neoplasm; NEN, neuroendocrine neoplasm; Adenoca., adenocarcinoma; Inadequate, inadequate sample for pathological diagnosis.

\section{Discussion}

Franseen needles have emerged as a preferable alternative to conventional needles as they can easily obtain larger tissue samples than conventional needles. For example, relative to a conventional needle, a Franseen needle of the same gauge provides approximately $5 \times$ the median area of tissue sample for histopathological evaluation [10]. In this context, larger samples preserve tissue architecture and can provide a more accurate and easier pathological diagnosis, relative to smaller samples. We have also reported that a macroscopic on-site evaluation of the macroscopically visible core can help determine the required number of needle punctures, even without rapid on-site cytological evaluation (ROSE) [7], which can reduce the burden on endosonographers and pathologists. Furthermore, obtaining a sufficient core sample can enable genetic analysis and molecular profiling $[8,14]$, which can contribute to personalized treatment selection. Several studies have already confirmed the efficacy of the Franseen needle [15-17]. A meta-analysis of 
15 studies with 1024 patients revealed that, relative to FNA needles and in the absence of ROSE, FNB needles provided better diagnostic adequacy for solid pancreatic lesions and required fewer needle passes to establish the diagnosis [18]. Our previous study also revealed that use of a Franseen needle provided sufficient tissue samples for histological interpretation in $96 \%$ of cases with a single needle pass and in $100 \%$ of cases with 3 needle passes [16].

Several studies have addressed factors that affect the diagnostic accuracy of EUS-FNA. Uehara et al. [19] reported that the diagnostic accuracy of EUS-FNA was not associated with lesion size, lesion location, or needle size, based on their retrospective study, and concluded that EUS-FNA was useful for diagnosing small pancreatic lesions $(<10 \mathrm{~mm})$. However, Agarwal et al. [20] reported that the diagnostic accuracy of EUS-FNA was lower for suspicious pancreatic lesions that were $<20 \mathrm{~mm}$, relative to lesions that were $>21 \mathrm{~mm}$. Haba et al. [21] also reported that a low diagnostic accuracy was independently associated with a nonpancreatic cancer final diagnosis, pancreatic head lesion location, lesion size of $<20 \mathrm{~mm}$, and absence of ROSE. Furthermore, they found that lesion size and ROSE had the greatest influence on diagnostic accuracy. Kurita et al. [22] also reported that the diagnostic accuracy of EUS-FNA was significantly lower for small pancreatic tumors $(<5 \mathrm{~mm})$ accompanied by chronic pancreatitis and pancreatic cancer. Thus, small lesion size appears to be associated with lower diagnostic accuracy, although those studies used conventional FNA needles and the pathological diagnosis was mainly based on cytological assessment. Therefore, it is unclear whether EUS-FNB using a Franseen needle is feasible for histopathological assessment of small pancreatic lesions.

The present study revealed that the overall accuracy of EUS-FNB (combining cytology and histology) was $98.7 \%$ for all lesions, and the accuracy was still high (94.3\%) for lesions that were $<10 \mathrm{~mm}$. Nevertheless, the diagnostic accuracy based on the histological examination alone was only $64.3 \%$ for small lesions $(<10 \mathrm{~mm})$, although the accuracy increased to $97.2 \%$ for lesions that were $>10 \mathrm{~mm}(p<0.001)$. In addition, lower diagnostic accuracy was associated with age $<70$ years and a non-pancreatic cancer final diagnosis, although these factors were not significant in the multivariate analysis, and only lesion size was an independent predictor of histopathological diagnosis accuracy. This may be related to cases with small lesions $(<10 \mathrm{~mm})$ often involving younger patients ( $<70$ years old) and patients with non-NEN tumors (Table 7).

A detailed examination of the EUS-FNB results for lesions that were $<10 \mathrm{~mm}$ revealed some interesting findings. First, sufficient samples could not be obtained for the histopathological diagnosis in all 4 cases that had lesions of $<5 \mathrm{~mm}$ although the histopathological diagnosis was accurate in 9 out of 10 cases (90\%) that had lesions measuring in the range of 6-10 mm. Thus, a lesion size of $<5 \mathrm{~mm}$, rather than $<10 \mathrm{~mm}$, may influence the accuracy of the histopathological diagnosis, which may be related to the difficulty of visualizing and puncturing these small lesions. Nevertheless, cytological diagnosis was possible in all 4 cases with lesion sizes of $<5 \mathrm{~mm}$, which would suggest that the needle punctured the lesion correctly. Therefore, we conclude that the histopathological diagnosis is likely more difficult than the cytopathological diagnosis in cases with such small lesions. However, combining the cytologic and histopathologic information provided by the biopsy may enhance the diagnostic accuracy in such cases.

Interestingly, all 4 cases with lesions of $<5 \mathrm{~mm}$ had a cytological diagnosis of NEN, which might have contributed to the failed histopathological diagnosis. However, the diagnostic accuracy of EUS-FNA is generally considered lower for pancreatic cancer than for other pathologies [22]. This may be because pancreatic cancer often involves an intense stromal desmoplastic reaction [23], which may make it difficult to aspirate the sample during FNB and to histopathologically diagnose the limited number of cancer cells in abundant desmoplastic tissue from a small specimen.

The present study has some limitations. First, the retrospective single-center study design and small number of patients are associated with risks of bias. However, during the study period, we routinely performed EUS-FNB with a Franseen needle for all patients 
with solid pancreatic masses, regardless of lesion size and location. Nevertheless, large prospective studies are needed to validate our findings. Second, the final diagnoses were confirmed based on the surgical specimen in 79 out of 159 patients, while the EUS-FNB findings and clinical course were used to confirm the diagnoses for the other 80 patients (73 malignant lesions and 7 benign lesions). It is possible that the 7 patients who were diagnosed with benign tumors might actually have had low-grade malignancy, although they did not exhibit any signs of malignancy during a $\geq 6$-month follow-up. Third, the study, including the assessment of diagnostic accuracy, is limited by a very small number of biopsies of lesions of $<5 \mathrm{~mm}$.

In conclusion, the diagnostic accuracy for small lesions ( $<10 \mathrm{~mm})$, especially for lesions of $<5 \mathrm{~mm}$, based on the histological examination alone, was significantly lower than for other lesions $(>10 \mathrm{~mm})$. Furthermore, multivariate analysis revealed that only lesion size was an independent predictor of histopathological diagnosis accuracy.

Author Contributions: Conceptualization, K.T. (Kosuke Takahashi)and I.Y.; methodology, K.T. (Kosuke Takahashi) and I.Y.; software, K.T. (Kosuke Takahashi); validation, K.T. (Kosuke Takahashi) and I.Y.; formal analysis, K.T.; investigation, K.T., T.H., Y.H., Y.A., and I.M.; resources, K.T. (Kosuke Takahashi), S.K., T.A., H.F., K.T. (Kazuto Tajiri), M.M., and T.T.; data curation, K.T. (Kosuke Takahashi); writing —original draft preparation, K.T. (Kosuke Takahashi); writing-review and editing, K.T. (Kosuke Takahashi) and I.Y.; visualization, K.T. (Kosuke Takahashi); supervision, I.Y.; project administration, I.Y.; funding acquisition, none. All authors have read and agreed to the published version of the manuscript.

Funding: This research received no external funding.

Institutional Review Board Statement: The study was conducted according to the guidelines of the Declaration of Helsinki, and approved by the Institutional Review Board of the institutional review board of our institution (R2020096, 25 August 2020).

Informed Consent Statement: Informed consent was obtained from all subjects involved in the study.

Data Availability Statement: No new data was created or analyzed in this study. Data sharing is not applicable to this article.

Conflicts of Interest: The authors declare no conflict of interest

\section{References}

1. Ngamruengphong, S.; Li, F.; Zhou, Y.; Chak, A.; Cooper, G.S.; Das, A. EUS and survival in patients with pancreatic cancer: A population-based study. Gastrointest. Endosc. 2010, 72, 78-83.e2. [CrossRef] [PubMed]

2. Othman, M.O.; Wallace, M.B. The role of endoscopic ultrasonography in the diagnosis and management of pancreatic cancer. Gastroenterol. Clin. N. Am. 2012, 41, 179-188. [CrossRef] [PubMed]

3. Yoshinaga, S.; Itoi, T.; Yamao, K.; Yasuda, I.; Irisawa, A.; Imaoka, H.; Tsuchiya, T.; Doi, S.; Yamabe, A.; Murakami, Y.; et al. Safety and efficacy of endoscopic ultrasound-guided fine needle aspiration for pancreatic masses: A prospective multicenter study. Dig. Endosc. 2020, 32, 114-126. [CrossRef] [PubMed]

4. Bang, J.Y.; Kirtane, S.; Krall, K.; Navaneethan, U.; Hasan, M.; Hawes, R.; Varadarajulu, S. In memoriam: Fine-needle aspiration, birth: Fine-needle biopsy: The changing trend in endoscopic ultrasound-guided tissue acquisition. Dig. Endosc. $2019,31,197-202$. [CrossRef] [PubMed]

5. Yasuda, I.; Goto, N.; Tsurumi, H.; Nakashima, M.; Doi, S.; Iwashita, T.; Kanemura, N.; Kasahara, S.; Adachi, S.; Hara, T.; et al. Endoscopic ultrasound-guided fine needle aspiration biopsy for diagnosis of lymphoproliferative disorders: Feasibility of immunohistological, flow cytometric, and cytogenetic assessments. Am. J. Gastroenterol. 2012, 107, 397-404. [CrossRef] [PubMed]

6. Iwashita, T.; Yasuda, I.; Doi, S.; Ando, N.; Nakashima, M.; Adachi, S.; Hirose, Y.; Mukai, T.; Iwata, K.; Tomita, E.; et al. Use of samples from endoscopic ultrasound-guided 19-gauge fine-needle aspiration in diagnosis of autoimmune pancreatitis. Clin. Gastroenterol. Hepatol. 2012, 10, 316-322. [CrossRef]

7. Iwashita, T.; Yasuda, I.; Mukai, T.; Doi, S.; Nakashima, M.; Uemura, S.; Mabuchi, M.; Shimizu, M.; Hatano, Y.; Hara, A.; et al. Macroscopic on-site quality evaluation of biopsy specimens to improve the diagnostic accuracy during EUS-guided FNA using a 19-gauge needle for solid lesions: A single-center prospective pilot study (MOSE study). Gastrointest. Endosc. 2015, 81, 177-185. [CrossRef]

8. Kovacevic, B.; Vilmann, P. EUS tissue acquisition: From A to B. Endosc. Ultrasound 2020, 9, 225-231. [CrossRef]

9. Adler, D.G.; Muthusamy, V.R.; Ehrlich, D.S.; Parasher, G.; Thosani, N.C.; Chen, A.; Buscaglia, J.M.; Appannagari, A.; Quintero, E.; Aslanian, H.; et al. A multicenter evaluation of a new EUS core biopsy needle: Experience in 200 patients. Endosc. Ultrasound 2019, 8, 99-104. [CrossRef] 
10. Mukai, S.; Itoi, T.; Yamaguchi, H.; Sofuni, A.; Tsuchiya, T.; Tanaka, R.; Tonozuka, R.; Honjo, M.; Fujita, M.; Yamamoto, K.; et al. A retrospective histological comparison of EUS-guided fine-needle biopsy using a novel franseen needle and a conventional end-cut type needle. Endosc. Ultrasound 2019, 8, 50-57. [CrossRef]

11. Bang, J.Y.; Hebert-Magee, S.; Hasan, M.K.; Navaneethan, U.; Hawes, R.; Varadarajulu, S. Endoscopic ultrasonography-guided biopsy using a Franseen needle design: Initial assessment. Dig. Endosc. 2017, 29, 338-346. [CrossRef] [PubMed]

12. Cotton, P.B.; Eisen, G.M.; Aabakken, L.; Baron, T.H.; Hutter, M.M.; Jacobson, B.C.; Mergener, K.; Nemcek, A., Jr.; Petersen, B.T.; Petrini, J.L.; et al. A lexicon for endoscopic adverse events: Report of an ASGE workshop. Gastrointest. Endosc 2010, 71, 446-454. [CrossRef] [PubMed]

13. Yasuda, I.; Iwashita, T.; Doi, S. Tips for endoscopic ultrasound-guided fine needle aspiration of various pancreatic lesions. J. Hepatobiliary Pancreat. Sci. 2014, 21, E29-E33. [CrossRef]

14. Elhanafi, S.; Mahmud, N.; Vergara, N.; Kochman, M.L.; Das, K.K.; Ginsberg, G.G.; Rajala, M.; Chandrasekhara, V. Comparison of endoscopic ultrasound tissue acquisition methods for genomic analysis of pancreatic cancer. J. Gastroenterol. Hepatol. 2019, 34, 907-913. [CrossRef]

15. Bang, J.Y.; Hebert-Magee, S.; Navaneethan, U.; Hasan, M.K.; Hawes, R.; Varadarajulu, S. EUS-guided fine needle biopsy of pancreatic masses can yield true histology. Gut 2018, 67, 2081-2084. [CrossRef]

16. Mita, N.; Iwashita, T.; Uemura, S.; Iwasa, Y.; Toda, K.; Mukai, T.; Miyazaki, T.; Yasuda, I.; Shimizu, M. Endoscopic UltrasoundGuided Fine Needle Biopsy Using 22-Gauge Franseen Needle for the Histological Diagnosis of Solid Lesions: A Multicenter Prospective Pilot Study. Dig. Dis. Sci. 2020, 65, 1155-1163. [CrossRef] [PubMed]

17. Fujita, A.; Ryozawa, S.; Mizuide, M.; Araki, R.; Nagata, K.; Tanisaka, Y.; Harada, M.; Ogawa, T.; Tashima, T.; Nonaka, K. Does endoscopic ultrasound-guided fine needle biopsy using a Franseen needle really offer high diagnostic accuracy? A propensity-matched analysis. Endosc. Int. Open 2019, 7, E1327-E1332. [CrossRef]

18. Khan, M.A.; Grimm, I.S.; Ali, B.; Nollan, R.; Tombazzi, C.; Ismail, M.K.; Baron, T.H. A meta-analysis of endoscopic ultrasoundfine-needle aspiration compared to endoscopic ultrasound-fine-needle biopsy: Diagnostic yield and the value of onsite cytopathological assessment. Endosc. Int. Open 2017, 5, E363-E375. [CrossRef]

19. Uehara, H.; Ikezawa, K.; Kawada, N.; Fukutake, N.; Katayama, K.; Takakura, R.; Takano, Y.; Ishikawa, O.; Takenaka, A. Diagnostic'accuracy of endoscopic ultrasound-guided fine needle aspiration for suspected pancreatic malignancy in relation to the size of lesions. J. Gastroenterol. Hepatol. 2011, 26, 1256-1261. [CrossRef]

20. Agarwal, B.; Abu-Hamda, E.; Molke, K.L.; Correa, A.M.; Ho, L. Endoscopic ultrasound-guided fine needle aspiration and multidetector spiral CT in the diagnosis of pancreatic cancer. Am. J. Gastroenterol. 2004, 99, 844-850. [CrossRef]

21. Haba, S.; Yamao, K.; Bhatia, V.; Mizuno, N.; Hara, K.; Hijioka, S.; Imaoka, H.; Niwa, Y.; Tajika, M.; Kondo, S.; et al. Diagnostic ability and factors affecting accuracy of endoscopic ultrasound-guided fine needle aspiration for pancreatic solid lesions: Japanese large single center experience. J. Gastroenterol. 2013, 48, 973-981. [CrossRef] [PubMed]

22. Kurita, Y.; Kuwahara, T.; Hara, K.; Mizuno, N.; Okuno, N.; Matsumoto, S.; Obata, M.; Koda, H.; Tajika, M.; Shimizu, Y.; et al. Features of chronic pancreatitis by endoscopic ultrasound influence the diagnostic accuracy of endoscopic ultrasound-guided fine-needle aspiration of small pancreatic lesions. Dig. Endosc. 2020, 32, 399-408. [CrossRef] [PubMed]

23. Kamisawa, T.; Wood, L.D.; Itoi, T.; Takaori, K. Pancreatic cancer. Lancet 2016, 388, 73-85. [CrossRef] 